\title{
On the Role of the Killing Tensor in the Einstein-Maxwell Theory
}

\author{
M. L. Woolley \\ Hove, Sussex, U.K.
}

Received April 6; in revised form June 1, 1973

\begin{abstract}
It is shown that when a four dimensional source-free Einstein-Maxwell space-time admits a group of motions leaving the electromagnetic field unchanged a linear relation exists between two Maxwell fields and the covariant derivative of a Killing vector. For the case in which the two electromagnetic fields are related by a duality rotation it is seen that a purely geometric form of Einstein's equations may be derived. The behaviour of these under a class of quasi conformal transformations of the metric is shown to lead to Harrison's theorem.
\end{abstract}

\section{Introduction}

While the geometric nature of the Einstein-Maxwell electromagnetic field is now well established (Rainich, 1925; Misner and Wheeler, 1957) there are nevertheless some aspects which are not very well understood. A particular case in point is the fact that solutions of the equations may be generated both from known solutions and from solutions of the empty-space equations $G_{\mu \nu}=0$ when a symmetry is present (Harrison, 1965 and 1968).

The object of the present paper is to establish a result which appears to be of relevance in explaining solution generation and the role which the Killing vector has in the transformation properties of the field equations.

\section{Einstein's Equations}

We shall consider a four dimensional space-time which satisfies the vacuum Einstein-Maxwell equations. These may be written

and

$$
\left.\begin{array}{c}
R_{\mu \nu}=4 \pi\left(F_{\mu \sigma} F_{v}^{\sigma}+{ }^{*} F_{\mu \sigma}{ }^{*} F_{v}^{\sigma}\right) \\
F^{\mu \nu}{ }_{\mid v}=0 \\
* F^{\mu v}{ }_{\mid v}=0
\end{array}\right\}
$$

where the vertical bar denotes covariant differentiation. 
The electromagnetic field is taken to be non-null so that

and

$$
\left.\begin{array}{rl}
F_{\mu \nu} F^{\mu v} & =0 \\
F_{\mu \nu}{ }^{*} F^{\mu v} & =0
\end{array}\right\}
$$

are not simultaneously satisfied.

We shall further assume that the space-time admits a group of motions so that Killing's equations

$$
\stackrel{\mathfrak{s}}{\mathfrak{v}} g_{\mu \nu}=v_{\mu \mid v}+v_{v \mid \mu}=0
$$

are integrable. These relations define the Killing tensor

$$
\xi_{\mu \nu}=v_{\mu \mid v} .
$$

For the present work we shall consider a non-null Killing vector $v_{\mu}$ which generates the invariance group of $F_{\mu v}$. That is, we must have

$$
\mathfrak{v} F_{\mu \nu}=0
$$

and the necessary and sufficient condition for this to be true is that a coordinate system can be found in which $F_{\mu \nu}$ is independent of one of the coordinates (Yano, 1955, p. 54).

With respect to the Killing vector $v_{\mu}$ the Lie derivatives of $F_{\mu \nu}$ and $F^{\mu v}$ take the forms

and

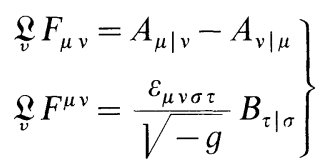

respectively where the vectors $A_{\mu}$ and $B_{\mu}$ are defined by

and

$$
\left.\begin{array}{l}
A_{\mu}=v^{v} F_{\mu \nu} \\
B_{\mu}=v^{v *} F_{\mu v}
\end{array}\right\}
$$

From (2.7) it follows that at least locally - depending on whether or not the space-time is simply connected - we may define scalar functions $\theta$ and $\varphi$ such that

$$
\left.\begin{array}{l}
A_{\mu}=\theta_{\mid \mu} \\
B_{\mu}=\varphi_{\mid \mu}
\end{array}\right\}
$$

These functions are essentially electromagnetic potentials.

We are now in a position to prove the following assertion:

In the four dimensional source-free Einstein-Maxwell theory the antisymmetric tensor

$$
\Gamma_{\mu \nu}=\xi_{\mu \nu}-4 \pi\left(\theta F_{\mu \nu}+\varphi^{*} F_{\mu \nu}\right)
$$

is a solution of Maxwell's equations. 
Proof. Taking the projection of the Ricci tensor in the direction of the Killing vector $v_{\mu}$ we obtain by means of Maxwell's equations

$$
R_{\mu \sigma} v^{\sigma}=4 \pi\left(\theta F_{\mu}^{\sigma}+\varphi^{*} F_{\mu}^{\sigma}\right)_{\mid \sigma} .
$$

But the Killing tensor $\xi_{\mu \nu}$ satisfies

$$
\xi_{\mid v}^{\mu v}=R^{\mu v} v_{v} .
$$

Hence, by Einstein's equations, we have

$$
\Gamma_{\mid v}^{\mu \nu}=0
$$

and $\Gamma_{\mu \nu}$ satisfies the first of Maxwell's equations (2.2).

On the other hand we have

$$
v_{\mu \mid v}=\frac{1}{2}\left(v_{\mu \mid v}-v_{v \mid \mu}\right)
$$

so the dual of the Killing tensor satisfies

$$
* \xi_{\mid v}^{\mu v}=0 \text {. }
$$

But in four dimensions the well-known identity

$$
{ }^{*} F_{\mu \sigma} F_{v}^{\sigma}=F_{\mu \sigma}{ }^{*} F_{v}^{\sigma}
$$

ensures that

$$
\left(\theta^{*} F^{\mu v}-\varphi F^{\mu v}\right)_{\mid v}=0 .
$$

Hence

$$
* \Gamma_{\mid v}^{\mu v}=0
$$

and the proof is complete.

With this result we have shown that the assumption of the existence of the invariant electromagnetic field $F_{\mu v}$, acting as the source of the space-time, carries with it in Einstein's theory the existence of the Maxwell field $\Gamma_{\mu \nu}$ related linearly to $F_{\mu \nu}$ by (2.10).

At this stage we will consider that $\Gamma_{\mu \nu}$, as well as $F_{\mu \nu}$, is a source of the space-time. On physical grounds this is not unreasonable since, if it does not vanish, $\Gamma_{\mu \nu}$ must have a non-trivial energy-momentum tensor

$$
T_{\mu \nu}=-\frac{1}{2}\left(\Gamma_{\mu \sigma} \Gamma_{v}^{\sigma}+{ }^{*} \Gamma_{\mu \sigma} * \Gamma_{v}^{\sigma}\right) .
$$

But the metric tensor $g_{\mu \nu}$ which determines $\Gamma_{\mu \nu}$ is the same as that which gives $F_{\mu \nu}$ and the Einstein tensor $G_{\mu \nu}$ is unique to within the cosmological term (Lovelock, 1971 and 1972). Thus, by Einstein's theory of gravitation, we must have

or

$$
G_{\mu v}=-8 \pi T_{\mu v}
$$

$$
R_{\mu \nu}=4 \pi\left(\Gamma_{\mu \sigma} \Gamma_{v}^{\sigma}+{ }^{*} \Gamma_{\mu \sigma}{ }^{*} \Gamma_{v}^{\sigma}\right) .
$$


However, for reasons which we shall discuss later, while it is certainly a sufficient condition on $\Gamma_{\mu \nu}$, that it satisfy (2.21), it would not in general appear to be a necessary one and for the remainder of our work here we shall consider the case in which $\Gamma_{\mu \nu}$ and $F_{\mu \nu}$ determine the same metric.

When (2.21) is true the theorem of Misner and Wheeler (1957) asserts that $F_{\mu \nu}$ and $\Gamma_{\mu \nu}$ must be related by a duality rotation. Thus there must exist a constant $\varepsilon$ such that

$$
\Gamma_{\mu \nu}=F_{\mu \nu} \operatorname{Cos} \varepsilon+{ }^{*} F_{\mu \nu} \operatorname{Sin} \varepsilon .
$$

From (2.10) we now see that

where

$$
v_{\mu \mid v}=a F_{\mu \nu}+b^{*} F_{\mu \nu}
$$

and

$$
\left.\begin{array}{l}
a=4 \pi \theta+\operatorname{Cos} \varepsilon \\
b=4 \pi \varphi+\operatorname{Sin} \varepsilon
\end{array}\right\}
$$

are electromagnetic potentials. If we define

$$
\lambda=v_{\sigma} v^{\sigma}
$$

then from (2.23) we obtain

$$
4 \pi\left(\lambda_{0}-\lambda\right)=a^{2}+b^{2}
$$

where $\lambda_{0}$ is a constant of integration. It now follows that

$$
F_{\mu \sigma} F_{v}^{\sigma}+{ }^{*} F_{\mu \sigma} * F_{v}^{\sigma}=\left(a^{2}+b^{2}\right)^{-1}\left(\xi_{\mu \sigma} \xi_{v}^{\sigma}+{ }^{*} \xi_{\mu \sigma} * \xi_{v}^{\sigma}\right)
$$

and we have shown that:

When $\Gamma_{\mu \nu}$ acts as a source of the space-time the Ricci tensor $R_{\mu \nu}$ is given by

$$
R_{\mu v}=\frac{\xi_{\mu \sigma} \xi_{v}^{\sigma}+* \xi_{\mu \sigma} * \xi_{v}^{\sigma}}{\lambda_{0}-\lambda}
$$

Regarding (2.28) as Einstein's equations we have a form which depends only on the metric tensor and Killing vector. Since these equations are sufficient to determine the metric tensor completely, in this case, we have formally proved the sufficiency of (2.22).

Finally it is worth remarking that while we have assumed $\Gamma_{\mu \nu}$ to be non-vanishing we have lost no generality since, when it is related to $F_{\mu \nu}$ by a duality rotation, it can be effectively transformed away by a gauge transformation.

For the present work we are interested in the implications which (2.28) has with regard to solution generation and we shall now look at this aspect in more detail. 


\section{Application to Solution Generation}

Perhaps one of the principal results in solution generation is that formally proved by Harrison (1965) to the effect that to each solution of the equations $G_{\mu \nu}=0$, which can be put in diagonal form, and which depends only on three variables, there exists a corresponding solution of the Einstein-Maxwell equations with an associated electromagnetic potential.

Let us consider a diagonal metric

$$
(d s)^{2}=\alpha\left(d x^{1}\right)^{2}+\beta\left(d x^{2}\right)^{2}+\gamma\left(d x^{3}\right)^{2}+\sigma\left(d x^{4}\right)^{2}
$$

for which the metric tensor satisfies

$$
G_{\mu \nu}=0
$$

and is independent of $x^{4}$. If we define the quantities

and

$$
\left.\begin{array}{l}
X=\alpha \sigma \\
Y=\beta \sigma \\
Z=\gamma \sigma
\end{array}\right\}
$$

the first six non-trivial components of the Einstein tensor $G_{\mu \nu}$ may be written:

$$
\begin{aligned}
& G_{11}=-\frac{\eta_{x}}{2}\left\{\left(\eta_{x} Z_{2}\right)_{2}+\left(\eta_{x} Y_{3}\right)_{3}\right\}+\frac{X_{2} Z_{2}+X_{3} Y_{3}-Y_{1} Z_{1}}{4 Y Z}+4 \pi E_{11} \\
& G_{12}=\frac{1}{2}\left\{\left(\frac{Z_{1}}{Z}\right)_{2}+\frac{Z_{1} Z_{2}}{2 Z^{2}}-\frac{Z_{1} X_{2}}{2 Z X}-\frac{Z_{2} Y_{1}}{2 Z Y}\right\}+4 \pi E_{12} \\
& G_{13}=\frac{1}{2}\left\{\left(\frac{Y_{1}}{Y}\right)_{3}+\frac{Y_{1} Y_{3}}{2 Y^{2}}-\frac{Y_{1} X_{3}}{2 Y X}-\frac{Z_{1} Y_{3}}{2 Z Y}\right\}+4 \pi E_{13} \\
& G_{22}=-\frac{\eta_{y}}{2}\left\{\left(\eta_{y} Z_{1}\right)_{1}+\left(\eta_{y} X_{3}\right)_{3}\right\}+\frac{Y_{1} Z_{1}+Y_{3} X_{3}-X_{2} Z_{2}}{4 X Z}+4 \pi E_{22} \\
& G_{23}=\frac{1}{2}\left\{\left(\frac{X_{2}}{X}\right)_{3}+\frac{X_{2} X_{3}}{2 X^{2}}-\frac{X_{2} Y_{3}}{2 X Y}-\frac{Z_{2} X_{3}}{2 Z X}\right\}+4 \pi E_{23} \\
& G_{33}=-\frac{\eta_{z}}{2}\left\{\left(\eta_{z} Y_{1}\right)_{1}+\left(\eta_{z} X_{2}\right)_{2}\right\}+\frac{Y_{1} Z_{1}+X_{2} Z_{2}-X_{3} Y_{3}}{4 X Y}+4 \pi E_{33}
\end{aligned}
$$

and, remembering that the curvature scalar $R$ vanishes for both of the cases which we are considering, we may write the last component $G_{44}$ as

$$
G_{44}=\frac{\sigma^{2}}{2 \sqrt{X Y Z}}\left\{\left(\frac{\sigma_{1}}{\sigma \eta_{x}}\right)_{1}+\left(\frac{\sigma_{2}}{\sigma \eta_{y}}\right)_{2}+\left(\frac{\sigma_{3}}{\sigma \eta_{z}}\right)_{3}\right\} \text {. }
$$


In the above relations the quantities $\eta_{x}, \eta_{y}$ and $\eta_{z}$ are defined by

and

$$
\left.\begin{array}{l}
\eta_{x}^{2}=\frac{X}{Y Z} \\
\eta_{y}^{2}=\frac{Y}{X Z} \\
\eta_{z}^{2}=\frac{Z}{X Y}
\end{array}\right\}
$$

respectively, $4 \pi E_{\mu \nu}$ is defined by

$$
4 \pi E_{\mu \nu}=-\frac{\xi_{\mu \sigma} \xi_{v}^{\sigma}+* \xi_{\mu \sigma} * \xi_{v}^{\sigma}}{\sigma}
$$

and subscripts 1,2 or 3 on $X, Y, Z$ and $\sigma$, and the brackets, denote differentiation with respect to the appropriate independent variable.

The Killing vector $v_{\mu}$ for the metric (3.1) has only one non zero component $v_{4}=\sigma$ and the energy-momentum tensor, in the presence of an electromagnetic field, calculated from the right hand side of (2.28), has the components given by

$$
\begin{aligned}
& -8 \pi T_{11}=\frac{-\alpha \lambda_{0}}{4 \sigma^{2}\left(\lambda_{0}-\sigma\right)}\left\{\frac{\sigma_{1}^{2}}{\alpha}-\frac{\sigma_{2}^{2}}{\beta}-\frac{\sigma_{3}^{2}}{\gamma}\right\}+4 \pi E_{11} \\
& -8 \pi T_{12}=\frac{-\sigma_{1} \sigma_{2} \lambda_{0}}{2 \sigma^{2}\left(\lambda_{0}-\sigma\right)}+4 \pi E_{12} \\
& -8 \pi T_{13}=\frac{-\sigma_{1} \sigma_{3} \lambda_{0}}{2 \sigma^{2}\left(\lambda_{0}-\sigma\right)}+4 \pi E_{13} \\
& -8 \pi T_{22}=\frac{-\beta \lambda_{0}}{4 \sigma^{2}\left(\lambda_{0}-\sigma\right)}\left\{-\frac{\sigma_{1}^{2}}{\alpha}+\frac{\sigma_{2}^{2}}{\beta}-\frac{\sigma_{3}^{2}}{\gamma}\right\}+4 \pi E_{22} \\
& -8 \pi T_{23}=\frac{-\sigma_{2} \sigma_{3} \lambda_{0}}{2 \sigma^{2}\left(\lambda_{0}-\sigma\right)}+4 \pi E_{23} \\
& -8 \pi T_{33}=\frac{-\gamma \lambda_{0}}{4 \sigma^{2}\left(\lambda_{0}-\sigma\right)}\left\{-\frac{\sigma_{1}^{2}}{\alpha}-\frac{\sigma_{2}^{2}}{\beta}+\frac{\sigma_{3}^{2}}{\gamma}\right\}+4 \pi E_{33} \\
& -8 \pi T_{44}=\frac{-1}{4\left(\lambda_{0}-\sigma\right)}\left\{\frac{\sigma_{1}^{2}}{\alpha}+\frac{\sigma_{2}^{2}}{\beta}+\frac{\sigma_{3}^{2}}{\gamma}\right\} .
\end{aligned}
$$

In addition, for the diagonal form (3.1), the potential functions $a$ and $b$, of (2.24), are found to be proportional and the electromagnetic potential $\theta$ 
is given by

$$
(4 \pi \theta+\operatorname{Cos} \varepsilon)^{2} c^{2}=\lambda_{0}-\sigma
$$

where $c$ is a constant.

If we first consider the empty space field satisfying (3.2) we see that the quantities $-4 \pi E_{m n}$, for $m$ and $n \in\{1,2,3\}$, may be equated to quantities which are unchanged in form by quasi conformal transformations of the form

$$
\left.\begin{array}{l}
\alpha \rightarrow \alpha \omega=\hat{\alpha} \\
\beta \rightarrow \beta \omega=\hat{\beta} \\
\gamma \rightarrow \gamma \omega=\hat{\gamma} \\
\sigma \rightarrow \sigma / \omega=\hat{\sigma} .
\end{array}\right\}
$$

On the other hand, in the presence of a vacuum electromagnetic field, these same quantities are equated to $\left(-8 \pi T_{m n}-4 \pi E_{m n}\right)$. Moreover, in the latter case, the last of Einstein's equations, corresponding to (3.10), may be written

$$
\left(\frac{\sigma_{1}}{\sigma \eta_{x} \sqrt{\lambda_{0}-\sigma}}\right)_{1}+\left(\frac{\sigma_{2}}{\sigma \eta_{y} \sqrt{\lambda_{0}-\sigma}}\right)_{2}+\left(\frac{\sigma_{3}}{\sigma \eta_{z} \sqrt{\lambda_{0}-\sigma}}\right)_{3}=0
$$

If we now suppose that $\{\hat{\alpha}, \hat{\beta}, \hat{\gamma}, \hat{\sigma}\}$ represents a solution of the Einstein-Maxwell equations while $\{\alpha, \beta, \gamma, \sigma\}$ satisfy $G_{\mu \nu}=0$ then the transformation (3.21) takes (3.22) into $G_{44}=0$ if $\omega$ is chosen so that

$$
\frac{\sqrt{\lambda_{0}}}{\hat{\sigma} \sqrt{\lambda_{0}-\hat{\sigma}}} \frac{\partial \hat{\sigma}}{\partial x^{n}}=\frac{1}{\sigma} \frac{\partial \sigma}{\partial x^{n}}, \quad n=1,2,3 .
$$

But this same condition ensures that the quantities $\left(-8 \pi \hat{T}_{m n}-4 \pi \hat{E}_{m n}\right)$ have the same form, as functions of $\sigma$, as $-4 \pi E_{m n}$, i.e. the equations

$$
\hat{G}_{\mu \nu}=-8 \pi \hat{T}_{\mu \nu}
$$

go over to just

$$
G_{\mu v}=0
$$

Thus we see that to the empty space metric $\{\alpha, \beta, \gamma, \sigma\}$ there corresponds the Einstein-Maxwell metric $\{\hat{\alpha}, \hat{\beta}, \hat{\gamma}, \hat{\sigma}\}$ and vice versa. This is Harrison's theorem. 
It is readily verified that $\omega$ is given by

$$
\omega=\frac{(\sigma+1)^{2}}{4 \lambda_{0}}
$$

while the electromagnetic potential, determined by $\hat{\sigma}$, is given by

$$
4 \pi \theta+\operatorname{Cos} \varepsilon=\frac{\sqrt{\lambda_{0}}}{c}\left(\frac{\sigma-1}{\sigma+1}\right) .
$$

These expressions agree exactly with those given by Harrison and we have seen here that they arise as a consequence of the geometrical form (2.28) of Einstein's equations.

Finally, if we consider the class of solutions for which $\lambda_{0}$ is zero, the following result is readily obtained:

The Einstein-Maxwell metric

$$
(d s)^{2}=\alpha\left(d x^{1}\right)^{2}+\beta\left(d x^{2}\right)^{2}+\gamma\left(d x^{3}\right)^{2}-\sigma\left(d x^{4}\right)^{2}
$$

determines a further Einstein-Maxwell metric

$$
(d s)^{2}=\omega\left[\alpha\left(d x^{1}\right)^{2}+\beta\left(d x^{2}\right)^{2}+\gamma\left(d x^{3}\right)^{2}\right]-\frac{\sigma}{\omega}\left(d x^{4}\right)^{2}
$$

by means of the equation

where

$$
\left(\frac{\omega^{v}}{\sigma \sqrt{\omega}}\right)_{\mid v}=0
$$

$$
\omega_{v}=\omega_{\mid v}
$$

and covariant differentiation is with respect to the metric tensor $g_{\mu v}$ $=\operatorname{Diagonal}(\alpha, \beta, \gamma,-\sigma)$. The electromagnetic potential for the first metric is given by

$$
(4 \pi \theta+\operatorname{Cos} \varepsilon)^{2} c^{2}=\sigma
$$

where $c$ is constant.

\section{Conclusions}

The relevance of the space-time symmetry to solution generation has been clearly indicated by the geometric form of Einstein's equations and the examples which we have just considered. However, in arriving at the Eqs. (2.28) we only established the sufficiency of the condition that the two electromagnetic fields $F_{\mu \nu}$ and $\Gamma_{\mu \nu}$ be related by a duality rotation. In determining whether this condition is necessary or not we might gain further information on the role played by the Killing vector. 
An approach which indicates that it is not necessary for the two fields to be related by a duality rotation lies in considering the relative orientations of the tetrads of eigenvectors which belong to the three tensors $\left(\xi_{\mu \sigma} \xi_{v}^{\sigma}+{ }^{*} \xi_{\mu \sigma} * \xi_{v}^{\sigma}\right),\left(F_{\mu \sigma} F_{v}^{\sigma}+{ }^{*} F_{\mu \sigma}{ }^{*} F_{v}^{\sigma}\right)$ and $\left(\Gamma_{\mu \sigma} \Gamma_{v}^{\sigma}+{ }^{*} \Gamma_{\mu \sigma}{ }^{*} \Gamma_{v}^{\sigma}\right)$.

For an arbitrary tensor of the form

$$
R_{\mu \nu}=4 \pi\left(F_{\mu \sigma} F_{v}^{\sigma}+{ }^{*} F_{\mu \sigma} * F_{v}^{\sigma}\right)
$$

where $F_{\mu \nu}$ is antisymmetric, the tensor

$$
f_{\mu \nu}=4 \sqrt{\pi}\left(F_{\mu \nu} \operatorname{Sin} \eta+{ }^{*} F_{\mu \nu} \operatorname{Cos} \eta\right)
$$

where

$$
\eta=\frac{\pi}{4}+\frac{1}{2} \operatorname{Tan}^{-1}\left(\frac{F_{\mu \nu} F^{\mu v}}{F_{\mu \nu}{ }^{*} F^{\mu \nu}}\right)
$$

is a projection operator on to one of its eigenvectors (Woolley, 1973). It follows that the linear relation (2.10) is equivalent to a relation between the above operators which may be associated with the three tensors $\xi_{\mu v}$, $F_{\mu v}$, and $\Gamma_{\mu v}$. If we take the dual relation into account we have two linear relations between eigenvectors. We may then use the linear independence of the eigenvectors as justification for taking one tetrad as a base set in a linear vector space - the eigenvectors of the other two tetrads being expanded in terms of these. When this is done we arrive at a fully determined system of algebraic equations for the rotation coefficients and the solution of these may be reduced to finding the roots of a quartic. The main conclusion which is reached is that the expansion of the eigenvectors associated with $\Gamma_{\mu \nu}$ in terms of those belonging to $F_{\mu \nu}$, is not, in general, one such that $\Gamma_{\mu \nu}$ and $F_{\mu \nu}$ need be related by a duality rotation, i.e. from purely algebraic considerations, $\Gamma_{\mu \nu}$ need not act as a source of the space time.

While the full meaning of the relation between the two electromagnetic fields remains to be studied it is apparent that the Killing tensor $v_{\mu \mid v}$ is related in quite a fundamental manner with the EinsteinMaxwell electromagnetic field.

Acknowledgements. I would like to give my thanks to Professor W. B. Bonnor, Head of the Mathematics Department at Queen Elizabeth College, University of London, and Dr. A. Held, for discussing this work with me. In this respect also I thank K. J. Whiteman of the Mathematics Department at the University of Sussex. 


\section{References}

1. Harrison, B. K.: Phys. Rev. 138, 2 B, B 488 (1965)

2. Harrison, B. K.: J. Math. Phys. 9, 1744 (1968)

3. Lovelock, D.: J. Math. Phys. 12, No. 3, 488 (1971)

4. Lovelock, D.: J. Math. Phys. 13, No. 6, 874 (1972)

5. Misner, C. W., Wheeler, J. A.: Annals of Phys. 2, 525 (1957)

6. Rainich, G. Y.: Trans. American Math. Soc. 27, 106 (1925)

7. Woolley, M.L.: On the eigenvectors of the energy-momentum tensor in the EinsteinMaxwell theory, to appear in Proc. Cambridge Philosophical Soc. 1973

8. Yano, K.: Theory of Lie derivatives and its applications. Amsterdam: North-Holland 1955

M. L. Woolley

96, Highdown Road

Hove BN 36 EA, Sussex, U.K. 